\title{
Myocarditis: a Whole Heart Involvement Revealed by Cardiac Magnetic Resonance Mapping. A Case- control Study
}

\section{Tiago Bartzen Pereira}

Hospital Moinhos de Vento

Maurício Balk

Hospital Moinhos de Vento

Gabriela Bartzen Pereira

Complexo Hospitalar Santa Casa de Porto Alegre: Santa Casa de Misericordia de Porto Alegre

\section{Victória Schmidt Ramos}

Hospital Moinhos de Vento

\section{Luciano Giordani}

Hospital Moinhos de Vento

Paulo Roberto Schvartzman

Hospital Moinhos de Vento

Luis Beck-da-Silva ( $\sim$ lbneto@hcpa.edu.br)

Hospital de Clinicas de Porto Alegre https://orcid.org/0000-0002-8095-1177

\section{Original Article}

Keywords: T1 mapping, T2 mapping, Late gadolinium enhancement, Myocarditis, Cardiac magnetic resonance

Posted Date: February 4th, 2021

DOI: https://doi.org/10.21203/rs.3.rs-174912/v1

License: (c) (i) This work is licensed under a Creative Commons Attribution 4.0 International License. Read Full License 


\section{Abstract}

Purpose: To ascertain if differences in T1 and T2 mapping exist between ventricular wall segments with and without late gadolinium enhancement (LGE) in patients with myocarditis, and to compare these findings to those of control subjects.

Methods: In a case-control design, 22 patients with Cardiac Magnetic Resonance (CMR) evidence of myocarditis and 18 controls with normal CMR were assessed. The workup included: (1) T1 mapping (ShMOLLI); (2) LGE; (3) T2 mapping (SSFP); and (4) the T2 signal intensity of the myocardium divided by that of skeletal muscle (T2 ratio). T1 and T2 mapping of affected (LGE+) and unaffected (LGE-) ventricular segments of cases were compared, as were those of controls versus cases.

Results: On within-group comparison, ventricular segments with evidence of late enhancement (LGE+) in cases showed a mean T1 value significantly different from that of unaffected (LGE-) ventricular walls (1057 \pm 30 vs. $1028 \pm 48 ; p=0.0001$ ). On between-group comparison, the mean T1 value of LGE- segments in cases was significantly different from the mean of the corresponding walls in controls $(1028 \pm 48 \mathrm{vs}$. $996 \pm 10 ; p<0.0001)$. The mean T2 maps of LGE- walls in cases were not statistically different from those of controls ( $49 \pm 4$ vs. $49 \pm 1 ; p=0.9229)$.

Conclusions: This case-control study suggests that T1 mapping demonstrates significant alterations in the myocardium of patients with myocarditis even in the absence of late gadolinium enhancement. Specifically, T1 mapping revealed diffuse myocardial involvement not evidenced by LGE imaging. T2 mapping was noncontributory.

\section{Introduction}

The current incidence of myocarditis is unknown. [1] The epidemiology of this condition is poorly documented, due to the heterogeneity of clinical presentation and challenging diagnosis. According to the Dallas criteria, myocarditis is defined histologically by the presence of an inflammatory infiltrate in the myocardium, alongside degenerative and/or necrotic changes in adjacent cardiomyocytes, which differ from the ischemic damage associated with myocardial infarction. [2] Etiologically, it may be secondary to infectious or noninfectious processes. In developed nations, the leading cause of myocarditis is viral infection, while in developing countries the main causes are rheumatic carditis, Chagas disease, and HIVrelated. [3] Among several methods available for diagnostic, cardiac magnetic resonance (CMR) is the noninvasive modality best able to characterize the inflamed myocardium, demonstrating edema, necrosis, and fibrosis. The Lake Louise Criteria (LLC) for diagnosis of myocarditis on CMR are based on techniques such as T2-weighted imaging, early gadolinium enhancement (EGE), and late gadolinium enhancement (LGE). However, these CMR sequences have some limitations, such as an inability to identify diffuse fibrosis and the need for paramagnetic contrast. Another CMR method, the T2 ratio, defined as the T2 signal intensity of the myocardium divided by that of skeletal muscle, has also been losing ground to newer, more objective imaging techniques. These include T1 mapping, contrast- 
enhanced T1-weighted imaging, characterization of the extracellular volume (ECV) fraction, and T2 mapping, all of which have demonstrated superior diagnostic accuracy compared to the LLC. Furthermore, T1 and T2 mapping do not require gadolinium contrast [4], while contrast-enhanced T1weighted imaging and ECV mapping do. The latter two techniques are now largely used in the diagnosis of cardiac amyloidosis, and will not be addressed in the present study. Considering advances in imaging modalities and to confirm the need for change in diagnostic criteria, a proposed update to the LLC was published in December 2018. [5] In view of the limitations of the current criteria for CMR diagnosis of myocarditis, and given of uncertainties surrounding the putative diagnostic superiority of T1 and T2 mapping, as well as the advantage of not requiring gadolinium contrast, we designed this study to test the hypothesis that T1 and T2 map values would be altered both within the myocardial wall segments affected by late gadolinium enhancement ( $(\mathrm{GE}+$ ) and in seemingly unaffected regions (LGE-). Within this context, the objective of the present case-control study is to compare T1 mapping, T2 mapping, and T2 ratio between LGE+ and LGE- wall segments in patients with myocarditis and controls without myocarditis.

\section{Methods}

\section{Study population profile}

This retrospective chart-review study included 22 cases with acute myocarditis (age $34 \pm 16$ years; $13 \%$ female) and 18 controls (age $42 \pm 12$ years; $16 \%$ female). Study participants underwent CMR at Hospital Moinhos de Vento, Brazil, between January 2017 and June 2019. Analysis of CMR reports was performed consecutively, based on the date of the scans. The criteria for inclusion of cases were presence of mesocardial and/or subepicardial LGE plus European Society of Cardiology (ESC) criteria for clinically suspected myocarditis. [6] All cases had (a) chest or abdominal pain, dyspnea, or palpitations; (b) elevation of cardiac troponin I or T levels $>0.16 \mu \mathrm{g} / \mathrm{L}$ (mean $6 \mu \mathrm{g} / \mathrm{L}, \mathrm{SD} 16 \mu \mathrm{g} / \mathrm{L}$ ); and (c) presence of LGE in the expected anatomical region on CMR. All controls presented with (a) chest pain, dyspnea, or palpitations; (b) normal/not available troponin I or troponin T values; and (c) no evidence of edema, necrosis, fibrosis, or ischemia on CMR. Cases and controls were matched by age and sex. The exclusion criteria were: CMR demonstrating LGE in an anatomic region suggestive of other conditions, such as ischemic cardiomyopathy, hypertrophic cardiomyopathy, idiopathic dilated cardiomyopathy, amyloidosis, sarcoidosis, aortic stenosis, or pulmonary hypertension; and contraindications to CMR.

In all patients, the workup included LGE (132 case wall segments and 108 control wall segments), T1 mapping (132 case wall segments and 108 control wall segments), T2 mapping (130 case wall segments and 108 control wall segments), and T2 ratio $\geq 2: 1$ ( 20 affected case wall segments and 21 unaffected case wall segments).

Other variables of interest were: reason for CMR; left ventricular ejection fraction; left ventricular dimensions (atrial, diastolic, and systolic) and volumes (end-diastolic volume, end-systolic volume, and stroke volume); and anatomic region of fibrosis (subepicardial or mesocardial; walls: anterior, inferior, 
posterior, lateral, or septal; and segments: basal, medial, and apical); comorbidities (ischemic heart disease, stroke, diabetes mellitus, hypertension, ventricular/supraventricular arrhythmia, smoking, renal failure, heart failure, neoplasia), presence of symptoms (dyspnea, chest pain/discomfort, palpitations, abdominal pain), troponin levels, and the endomyocardial biopsy report.

Ventricular walls were analyzed according to the presence or absence of LGE. The T1 and T2 maps of the affected (LGE+) walls of cases were compared with the T1 and T2 maps of the contralateral unaffected (LGE-) walls of the same patients. In addition, the T1 and T2 maps of the LGE- walls of cases were compared with the T1 and T2 maps of the same walls in non-myocarditis controls. The mean T1 and T2 values of the LGE+ walls, the mean T1 and T2 values of the LGE- walls, and the mean T1 and T2 values of controls were compared. Figure 1 shows image analysis among myocardial walls.

For analysis of T1 and T2 maps, the average values obtained in controls were considered the reference range for normality.

Ethical approval was granted for all study procedures. As the study was purely observational, there were no physical or biological risks. There was also no personal contact with the participants, nor via telephone or social media. Data analysis was confidential, and the participants' names, addresses, and other contact information were not disclosed. In view of the foregoing and of the impossibility of accessing the participants' contact information in medical records, pursuant to National Health Council Resolution 466/12, the institutional Research Ethics Committee waived the usual informed consent requirement.

\section{Cardiovascular magnetic resonance}

CMR imaging was performed at 1.5 Tesla in a Siemens Healthcare model AERA 45mT scanner, using an 18-channel coil. Briefly, cine images were obtained in three long-axis sections (four-chamber, threechamber, two-chamber) and in the short-axis plane, from the base to the apex of the heart. Tissue characterization was performed in a single mid-ventricular short-axis view of the left ventricle, with T1 and T2 mapping, turbo spin-echo (TSE) T2-weighted, and short tau inversion recovery (STIR) sequences. LGE images were acquired by sectioning the whole heart, in a manner similar to the cine acquisition along the same axis. For T1 mapping, the shortened modified Look-Locker Inversion recovery (ShMOLLI) acquisition method was used before the administration of contrast agent. LGE images were acquired in the long- and short=axis planes, using a T1-weighted phase-sensitive inversion recovery (PSIR) sequence, 10 minutes after intravenous administration of gadobutrol (Gadovist - Bayer, total $0.20 \mathrm{mmol} / \mathrm{kg}$ ).

\section{Image analysis}

Left ventricular ejection fraction was analyzed on cine images using Argus software (Siemens Medical Solutions). Short-axis T1 and T2 mapping images, T2 ratio, and LGE were then analyzed. On T2-weighted dark blood images, edema is diagnosed when the T2 ratio is $\geq 2: 1$. The ratio was obtained as the T2 signal intensity of the myocardial region of interest with altered LGE divided by the signal intensity of 
skeletal muscle. T1 and T2 values were obtained from quantitative analysis of all T1 and T2 maps, rather than on visualization of color mapping. When delimiting the endo- and epicardial contours, care was taken to avoid contamination by the ventricular cavity and extramyocardial structures to minimize the partial volume effect on T1/T2 values of the myocardium. In acute myocarditis, identification of remote myocardium can be challenging, because the inflammatory process is often global; thus, a myocardial region without LGE was chosen to represent the myocardium least affected by the disease process, with care taken to also avoid regions of abnormally low signal intensity. Acute myocardial inflammation was diagnosed when the T1 value was $>996 \mathrm{~ms}$ and the T2 value was $>49 \mathrm{~ms}$. Focal areas of LGE were defined as those with a standard deviation of signal intensity $(\mathrm{SI}) \geq 2.0$ above the mean $\mathrm{SI}$ of the remote myocardium. To calculate the extent of myocardial injury detected by tissue characterization techniques, the percentage of abnormal myocardium, as defined above, was determined for each segment and then averaged for that patient.

\section{Assessment of CMR image quality}

Each myocardial segment of the left ventricle was rigorously assessed for image quality before inclusion in the final analyses. Only segments with minimal or no artifacts were included. Three controls were rejected due to artifacts. Four controls were excluded due to unavailability of map data.

\section{Statistical analysis}

Data were expressed as mean \pm standard deviation or median (confidence interval). Student's t-test was used for analysis of normally distributed continuous variables. For non-normal continuous variables, the nonparametric Wilcoxon-Mann-Whitney test was used. For categorical variables, the chi-square or Fisher's exact tests were used. The significance level was set at $5 \%$. Analyses were carried out in the SPSS 21.0 (SPSS, Chicago, IL) and MedCalc 2020 software environments. Due to the unclear prevalence of myocarditis, sample size calculation was not done. The initial sampling plan provided for 20 cases of myocarditis; 22 cases were found and ultimately included. This number is consistent with the existing literature. [7]

\section{Results}

The most common symptom reported as the reason for suspicion of myocarditis and performance of CMR was chest pain (91\%). The included patients had few comorbidities. The most prevalent was hypertension ( $18 \%$ of cases and $11 \%$ of controls). Almost $70 \%$ of cases underwent CMR as hospital inpatients, while $95 \%$ of controls were scanned in an outpatient setting. Structurally, case and control hearts were very similar; however, cases had a lower left ventricular ejection fraction (LVEF) than controls $(51 \pm 6 \%$ vs $65 \pm 6 \% ; p<0.0001)$, as well as smaller stroke volumes ( $84 \pm 21$ vs $102 \pm 18 \mathrm{~mL} ; p<0.0079)$. Only one patient with myocarditis underwent biopsy $(5 \%)$. The clinical and anatomical characteristics of the patients, stratified by group, are given in Table 1 .

\section{T2 ratio}


For analysis of the $\mathrm{T} 2$ ratio, values $\geq 2: 1$ were considered abnormal.

The mean T2 ratio for LGE+ regions in cases was $2.75 \pm 1$, which is indicative of myocardial wall edema.

The mean $T 2$ ratio for LGE- regions was $1.50 \pm 0.2$, which represents a normal value for these walls.

Accordingly, comparison of the $\mathrm{T} 2$ ratio in affected vs unaffected walls showed a statistically significant difference ( $2.75 \pm 1$ vs $1.50 \pm 0.2 ; p<0.0001)$.

\section{Late gadolinium enhancement (LGE)}

In patients with myocarditis, $L G E+$ images were often seen in more than one ventricular wall segment. The most commonly affected region was the posterior wall (95\%), followed by the lateral wall (68\%), anterior wall (32\%), inferior wall (32\%), and septum (18\%). Regarding myocardial injury pattern, mesocardial involvement was most common ( $86 \%)$, followed by the subepicardium (77\%). The mean fibrosis mass was $12 \mathrm{~g}$ ( $9 \%$ of the myocardium). The number of segments affected by LGE was 58 of 132 in cases $(44 \%)$ and 0 of $108(0 \%)$ in controls.

\section{T1 mapping}

On analysis of T1 mapping, values $\geq 996 \mathrm{~ms}$ were considered abnormal. By this parameter, the number of affected segments was 111 of $132(84 \%)$ in cases. The LGE+ segments of the cases showed a mean T1 value significantly different from the LGE- segments of the same patients $(1057 \pm 30$ vs $1028 \pm 48$; $p=0.0001)$. On between-group comparison, the mean T1 maps of LGE- ventricular walls in cases were significantly different from the mean of the corresponding walls in controls $(1028 \pm 48 \mathrm{vs.} 996 \pm 10$; $p<0.0001)$. The mean T1 values in each group are given in Table 2.

Observing the most frequently abnormal region in our patient population, the posterior wall, the mean T1 value of the affected segment in cases was $1068 \pm 47$ ms significantly different from all unaffected contralateral segments in these cases same $(1027 \pm 49 \mathrm{~ms}, \mathrm{p}=0.0011)$. This change remained significant when we compared all unaffected segments of cases to those of controls $(1027 \pm 49$ vs $994 \pm 38 \mathrm{~ms}$, $p=0.0084)$. Figure 2 shows a representative image of a posterior wall.

\section{T2 mapping}

On analysis of T2 mapping, values $\geq 49 \mathrm{~ms}$ were considered abnormal. By this parameter, the number of affected segments was 69 of $130(53 \%)$ in cases. The LGE+ segments of the cases showed a mean T2 value significantly different from the LGE- segments of the same patients $(51 \pm 2$ vs $49 \pm 4 ; p=0.0008)$. The mean T2 values of LGE- segments among cases were not significantly different from the mean T2 values of controls ( $49 \pm 4$ vs $49 \pm 1 ; p=0.9229)$. The mean T2 values in each group are given in Table 3 .

Regarding the posterior wall, T2 values again showed a significant difference between abnormal myocardial segments and unaffected walls in the same patients ( $52 \pm 5$ vs $49 \pm 4 \mathrm{~ms} ; \mathrm{p}=0.0062)$. Figure 3 
shows a representative image of a posterior wall and Figure 4 shows the relationship among different methods of image.

\section{Discussion}

The present case-control study demonstrates that T1 mapping allows a more comprehensive, in-depth assessment of supposedly normal myocardium in patients with CMR-proven myocarditis. Comparison of LGE+ versus LGE- segments in cases and of cases versus controls revealed not only a regional inflammatory process, but also diffuse myocardial involvement.

Although T1 mapping has been progressively used as an adjunctive tool in the diagnosis of myocarditis, [8] the present study was designed to investigate this method as a means of detecting myocardial involvement in areas of the heart that are considered unaffected by myocarditis when evaluated by LGE alone. Comparatively, we found that even seemingly normal myocardial segments in patients with myocarditis are indeed also compromised by inflammation. A mean T1 value of $1028 \pm 48 \mathrm{~ms}$ in the unaffected walls of the cases proved to be statistically different from that of controls, which confirms the hypothesis that "unaffected" segments were in fact not normal. On the other hand, myocardial edema, as assessed by T2 mapping, showed no difference between the LGE- segments of cases and those of controls. The findings of this study are consistent with the existing literature. [9]

The proportion of affected segments in cases was $44 \%$ when analyzed by LGE alone and $84 \%$ when assessed by T1 mapping. This result was interpreted as demonstrating a significant diffuse involvement of the myocardium, to the extent that almost the entire heart could be considered impaired in our patients with myocarditis.

The contribution of this finding to our knowledge of myocarditis is twofold: a) by enhancing the diagnostic performance of CMR in patients with myocarditis, particularly in borderline or difficult-todiagnose cases; and $b$ ) by introducing a novel concept in the diagnosis of myocarditis which allows objective, numerical, and quantifiable assessment of myocardial involvement, unlike current LGE-based criteria, in which the diagnosis is subjective and operator-dependent. In addition, it should be noted that T1 mapping obviates the use of gadolinium-based contrast agents, which eliminates the risk of allergic reactions, allows use in patients with renal failure, and reduces cost. The First Brazilian Guideline on Myocarditis (1) does not mention this imaging method, and the Second Guideline on Cardiac Magnetic Resonance [10] only notes T1/T2 mapping as a "promising" technique.

A multicenter observational study showed that T1 and ECV values were strong predictors of poor prognosis in non-ischemic dilated cardiomyopathy. [11] Nevertheless, whether this altered myocardium is in itself a predictor of cardiovascular events in myocarditis and other cardiovascular diseases remains unknown, due to a lack of studies with sufficient follow-up.

The diffuse $\mathrm{T} 1$ abnormalities in seemingly unaffected myocardial segments described in our study may have a major prognostic impact in the long term. Taylor et al. note that diffuse fibrosis has been identified 
as an etiological factor in diastolic dysfunction, heart failure, and sudden death. [12]

Regarding T2 mapping, some studies have shown that this method might be able to locate areas of involved in myocarditis with better sensitivity than conventional T2-weighted images alone. In 1.5-Tesla CMR, a $>59$-ms cutoff value demonstrated $94 \%$ sensitivity and $97 \%$ specificity for identification of affected myocardium. [13] Using a 49-ms threshold, our study confirmed a significant difference between cases with affected LGE+ walls and controls, but was unable to individually differentiate LGE+ and LGEsegments within cases (Table 3 ).

The mean T2 ratio in LGE+ segments was $2.75 \pm 1$, an abnormal value, whereas in segments without late gadolinium enhancement (LGE-) this ratio was normal. While T1 mapping was able to demonstrate that, in addition to a regional inflammatory process, myocarditis is characterized by widespread, diffuse myocardial involvement, the $\mathrm{T} 2$ ratio was consistent with the presence or absence of late gadolinium enhancement and did not reflect this diffuse inflammation. In short, the T2 ratio failed to detect the alterations suggestive of diffuse involvement detected by T1 mapping.

In short, T1 mapping was the only CMR technique capable of identifying diffuse changes in myocardial tissue, demonstrating abnormalities even in seemingly normal ventricular walls.

\section{Study Limitations}

Patients included in this study were selected at the time of CMR and not necessarily at the time of diagnosis of myocarditis. This may have somewhat reduced the diagnostic accuracy of CMR. However, as our main objective was to compare myocardial segments in the same patients, this limitation may actually have enhanced rather than jeopardized our analysis.

All controls had absence of late gadolinium enhancement and higher T1 values than reported in previous studies. [14] This may have reduced the odds of finding significant differences between cases and controls. However, even considering this unexpected finding, we were able to detect a significant difference between the T1 values of controls and the LGE- segments of cases. We thus believe this was a conservative bias. This limitation may also have decreased the statistical significance of the analysis of T2 map values between groups.

\section{Conclusion}

This study suggests that, in patients with myocarditis, even ventricular wall segments with no LGE are abnormal on T1 mapping. The altered T1 map values found in LGE- were intermediate between those of LGE+ walls in cases and those of LGE- walls in controls. Specifically, T1 mapping revealed a diffuse myocardial involvement not evidenced by LGE imaging. This method could be used to improve the accuracy of CMR imaging in the diagnosis of myocarditis.

\section{Abbreviations}


CMR, cardiac magnetic resonance

ECV, extracellular volume

EGE, early gadolinium enhancement

ESC, European Society of Cardiology

LGE, late gadolinium enhancement

LLC, Lake Louise Criteria

LVEF, lower left ventricular ejection fraction

PSIR, phase-sensitive inversion recovery

ShMOLLI, shortened modified Look-Locker Inversion recovery

SI, signal intensity

STIR, short tau inversion recovery

TSE, turbo spin-echo

\section{Declarations}

\section{Funding}

No funding was received for the preparation of this work.

\section{Author contributions}

All authors contributed to the study conception and design. Material preparation, data collection and analysis were performed by Tiago Bartzen Pereira, Luis Beck-da-Silva e Paulo Roberto Schvartzman. The first draft of the manuscript was written by Tiago Bartzen Pereira and all authors commented on previous versions of the manuscript. All authors read and approved the final manuscript.

\section{Compliance with ethical standards}

\section{Conflict of Interest}

The authors declare that they have no conflict of interest.

\section{Ethical approval}

All procedures performed in the present study were in accordance with the ethical standards of the institutional research committee and with the 1964 Helsinki declaration and its later amendments or 
comparable ethical standards.

\section{Informed consent}

As the study was purely observational, there were no physical or biological risks. There was also no personal contact with the participants, nor via telephone or social media. Data analysis was confidential, and the participants' names, addresses, and other contact information were not disclosed. In view of the foregoing and of the impossibility of accessing the participants contact information in medical records, the institutional Research Ethics Committee waived the usual informed consent requirement.

\section{Declarations:}

Not applicable

\section{References}

1. Montera MW, Mesquita ET, Colafranceschi AS, et al. I Brazilian guidelines on myocarditis and pericarditis. Arq Bras Cardiol 2013;100(4 Suppl 1):1-36. doi: 10.5935/abc.2013S004.

2. Aretz HT, Billingham ME, Edwards WD, et al. Myocarditis. A histopathologic definition and classification. Am J Cardiovasc Pathol 1987;1:3-14.

3. Mahrholdt H, Wagner A, Deluigi CC, et al. Presentation, patterns of myocardial damage, and clinical course of viral myocarditis. Circulation 2006;114:1581-90. doi:

10.1161/CIRCULATIONAHA.105.606509.

4. Bohnen S, Radunski UK, Lund GK, et al. Performance of t1 and t2 mapping cardiovascular magnetic resonance to detect active myocarditis in patients with recent-onset heart failure. Circ Cardiovasc Imaging 2015;8:e003073. doi: 10.1161/CIRCIMAGING.114.003073.

5. Ferreira VM, Schulz-Menger J, Holmvang G, et al. Cardiovascular Magnetic Resonance in Nonischemic Myocardial Inflammation: Expert Recommendations. J Am Coll Cardiol

2018;72:3158-76. doi: 10.1016/j.jacc.2018.09.072.

6. Caforio AL, Pankuweit S, Arbustini E, et al. Current state of knowledge on aetiology, diagnosis, management, and therapy of myocarditis: a position statement of the European

Society of Cardiology Working Group on Myocardial and Pericardial Diseases. Eur Heart J 2013;34:263648d. doi: 10.1093/eurheartj/eht210.

7. Dabir D, Vollbrecht TM, Luetkens JA, et al. Multiparametric cardiovascular magnetic resonance imaging in acute myocarditis: a comparison of different measurement approaches. J Cardiovasc Magn Reson 2019;21:54. doi: 10.1186/s12968-019-0568-x.

8. Lurz P, Luecke C, Eitel I, et al. Comprehensive Cardiac Magnetic Resonance Imaging in Patients With Suspected Myocarditis: The MyoRacer-Trial. J Am Coll Cardiol 2016;67:1800-

11. doi: 10.1016/j.jacc.2016.02.013.

9. Ferreira VM, Piechnik SK, Dall'Armellina E, et al. T(1) mapping for the diagnosis of acute myocarditis using CMR: comparison to T2-weighted and late gadolinium enhanced imaging.

JACC Cardiovasc Imaging 2013;6:1048-58. doi: 10.1016/j.jcmg.2013.03.008. 
10. Sara L, Szarf G, Tachibana A, et al. II Diretriz de Ressonância Magnética e Tomografia Computadorizada Cardiovascular da Sociedade Brasileira de Cardiologia e do Colégio Brasileiro de Radiologia [II Guidelines on Cardiovascular Magnetic Resonance and Computed Tomography of the Brazilian Society of Cardiology and the Brazilian College of Radiology]. Arq Bras Cardiol 2014;103(6 Suppl 3):1-86. doi: 10.5935/abc.2014S006

11. Puntmann VO, Carr-White G, Jabbour A, et al. T1-Mapping and Outcome in Nonischemic Cardiomyopathy: All-Cause Mortality and Heart Failure. JACC Cardiovasc Imaging. 2016;9(1):40-50. doi: 10.1016/j.jcmg.2015.12.001. Erratum in: JACC Cardiovasc Imaging. 2017;10(3):384.

12. Taylor AJ, Salerno M, Dharmakumar R, Jerosch-Herold M. T1 Mapping: Basic Techniques and Clinical Applications. JACC Cardiovasc Imaging 2016;9:67-81. doi: 10.1016/j.jcmg.2015.11.005

13. Thavendiranathan $P$, Walls $M$, Giri S, et al. Improved detection of myocardial involvement in acute inflammatory cardiomyopathies using T2 mapping. Circ Cardiovasc Imaging 2012;5:102-10. doi: 10.1161/CIRCIMAGING.111.967836.

14. Ferreira VM, Piechnik SK, Dall'Armellina E, et al. Native T1-mapping detects the location, extent and patterns of acute myocarditis without the need for gadolinium contrast agents. J Cardiovasc Magn Reson 2014;16:36. doi: 10.1186/1532-429X-16-36.

\section{Tables}

Tables 1-3 are available in the Supplemental Files

\section{Figures}




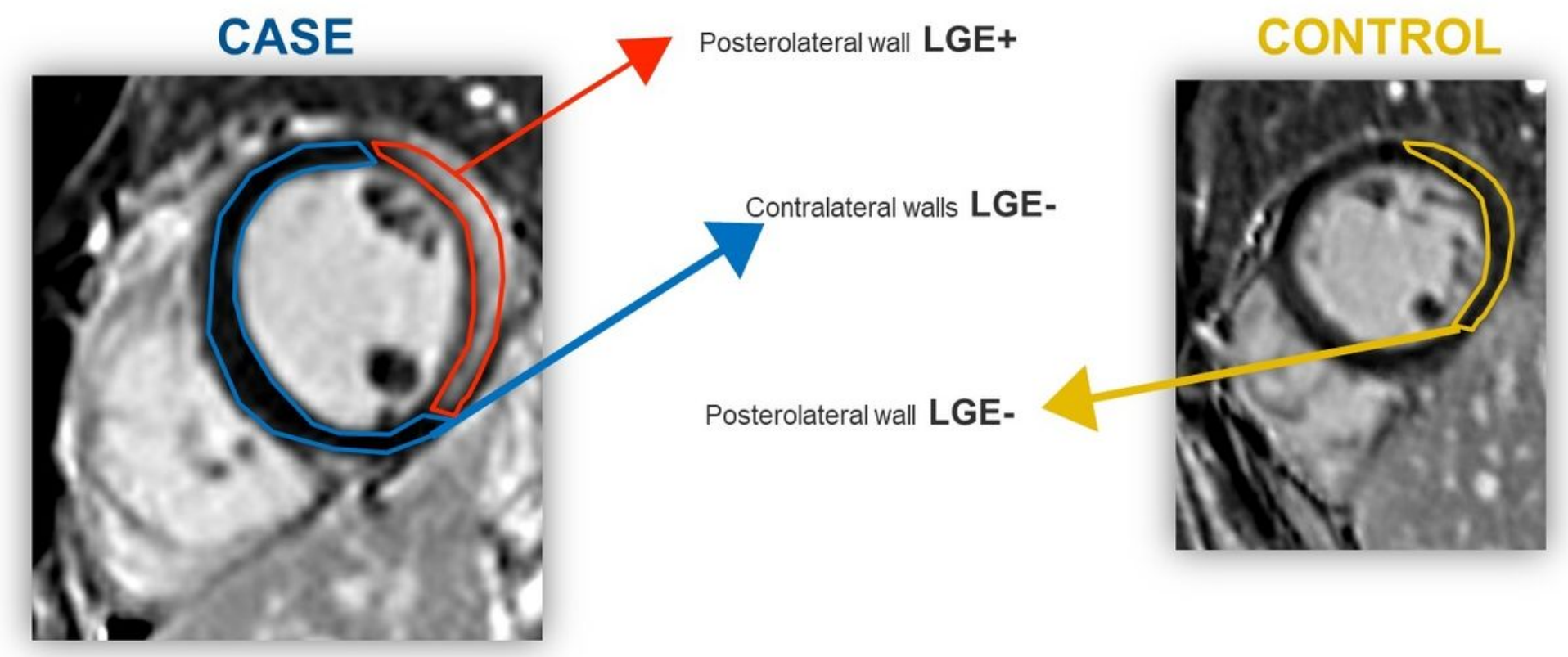

Figure 1

shows image analysis among myocardial walls. 


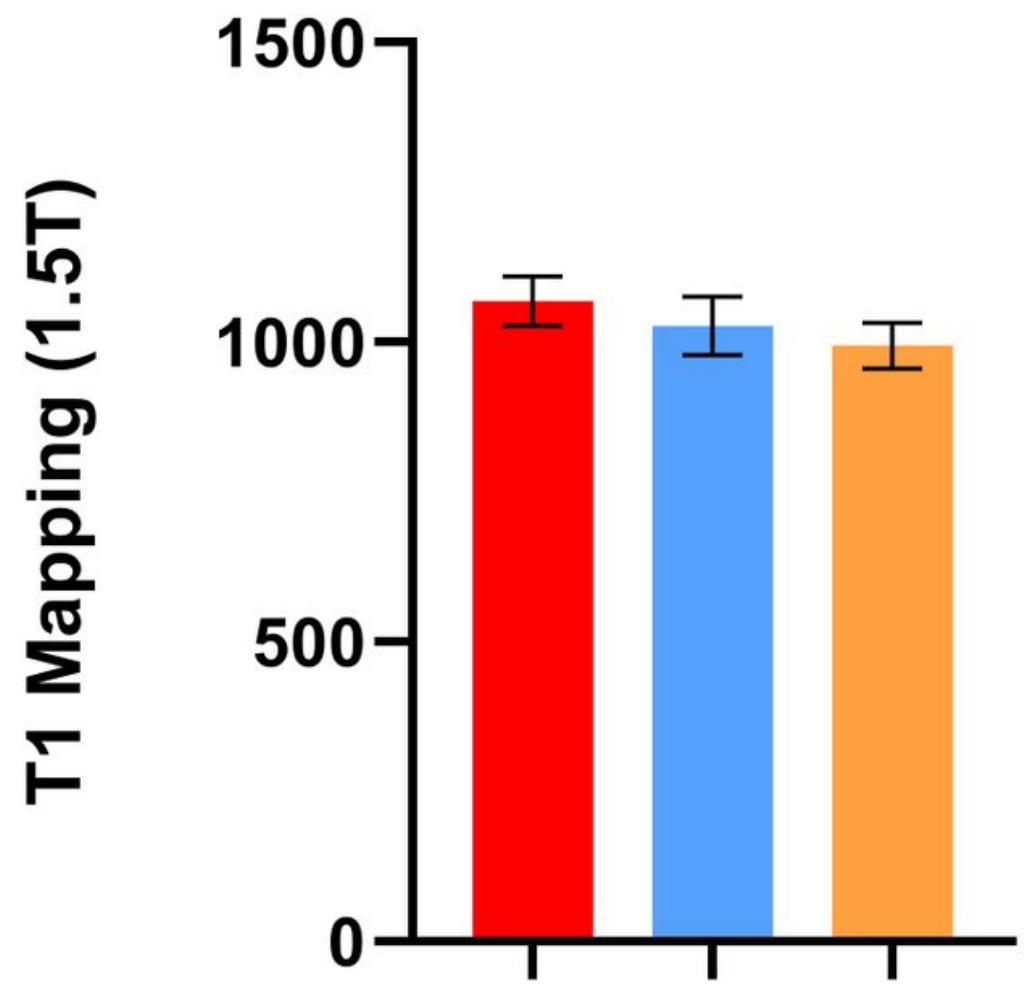

- Case LGE + Case LGE Control LGE -

Figure 2

shows a representative image of a posterior wall. 


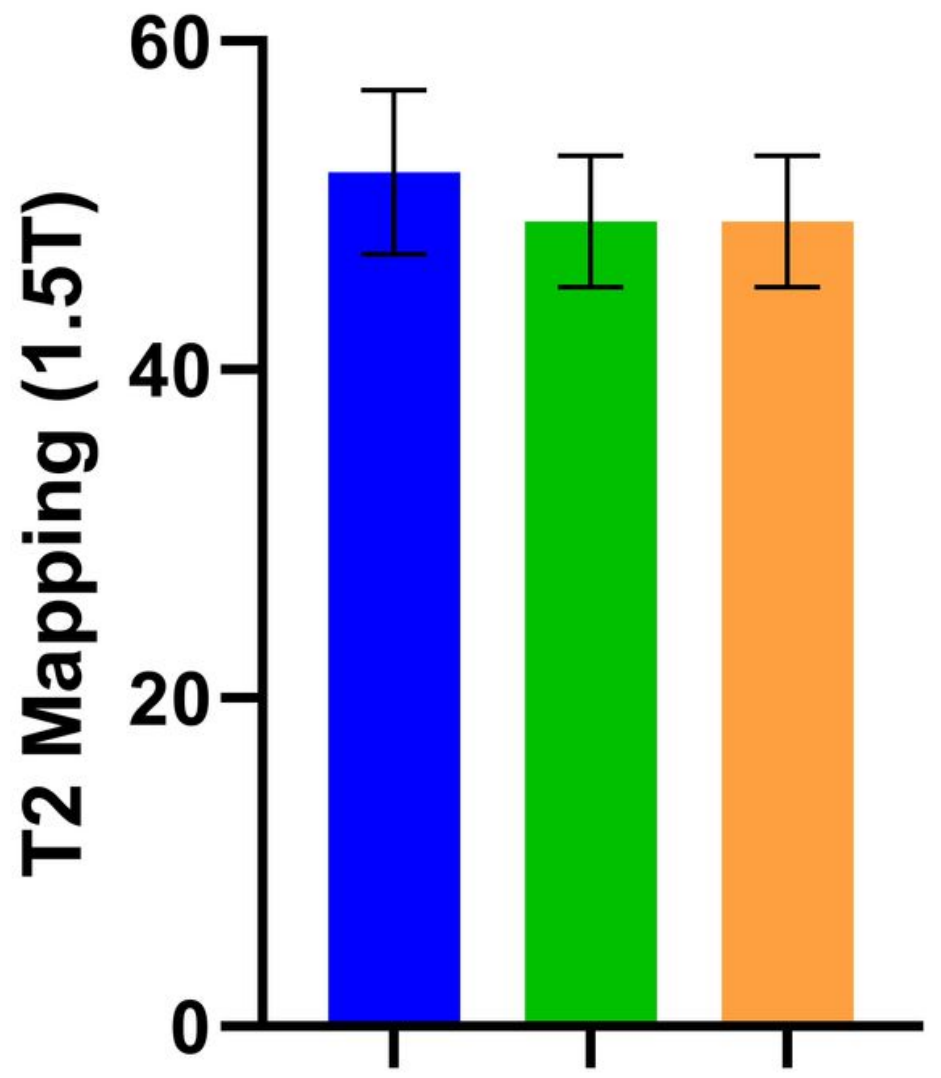

Case LGE + Case LGE Control LGE -

Figure 3

shows a representative image of a posterior wall 

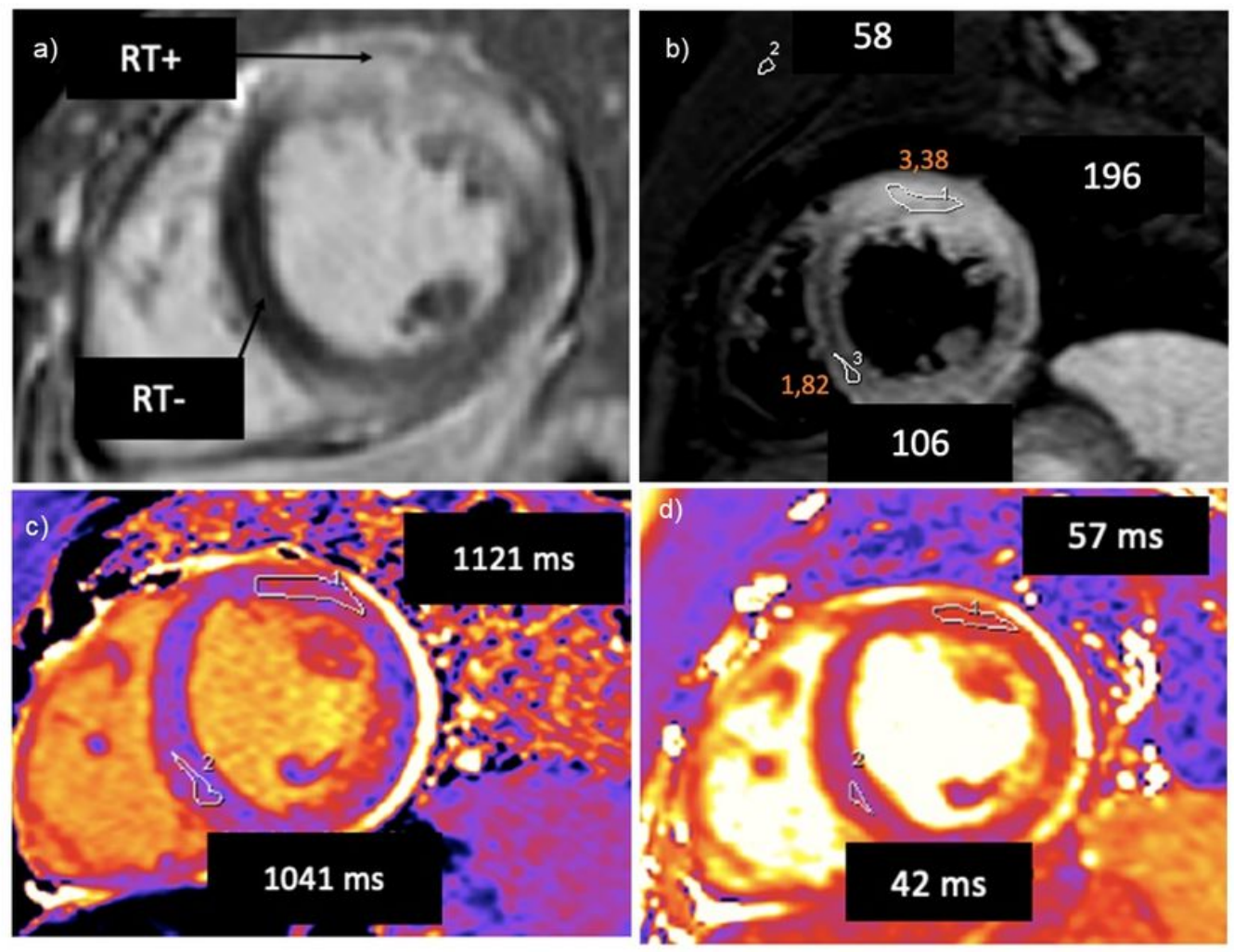

Figure 4

shows the relationship among different methods of image.

\section{Supplementary Files}

This is a list of supplementary files associated with this preprint. Click to download.

- Table1.pdf

- Table2.pdf

- Table3.pdf 